OPEN ACCESS

Edited by: Reza Lashgari,

Shahid Beheshti University, Iran

Reviewed by:

Antonio Lalueza,

Hospital Universitario 12 de

Octubre, Spain

Mohsen Rokni,

Tehran University of Medical

Sciences, Iran

*Correspondence:

Varut Vardhanabhut

varv@hku.hk

Specialty section:

This article was submitted to

Pulmonary Medicine,

a section of the journal

Frontiers in Medicine

Received: 26 August 2021 Accepted: 27 December 2021 Published: 24 February 2022

Citation:

Lau K-Y, Ng K-S, Kwok K-W, Tsia K-M, Sin C-F, Lam C-W and

Vardhanabhuti $V$ (2022) An Unsupervised Machine Learning

Clustering and Prediction of Differential Clinical Phenotypes of COVID-19 Patients Based on Blood

Tests - A Hong Kong Population

Study. Front. Med. 8:764934

doi: 10.3389/fmed.2021.764934

\section{An Unsupervised Machine Learning Clustering and Prediction of Differential Clinical Phenotypes of COVID-19 Patients Based on Blood Tests - A Hong Kong Population Study}

\author{
Kitty Yu-Yeung Lau ${ }^{1}$, Kei-Shing $\mathrm{Ng}^{2}$, Ka-Wai Kwok ${ }^{3}$, Kevin Kin-Man Tsia ${ }^{4}$, \\ Chun-Fung Sin $^{5}$, Ching-Wan Lam ${ }^{5}$ and Varut Vardhanabhuti ${ }^{2 \star}$
}

${ }^{1}$ Biomedical Engineering Programme, The University of Hong Kong, Hong Kong, Hong Kong SAR, China, ${ }^{2}$ Department of Diagnostic Radiology, Li Ka Shing Faculty of Medicine, The University of Hong Kong, Hong Kong, Hong Kong SAR, China, ${ }^{3}$ Department of Mechanical Engineering, The University of Hong Kong, Hong Kong, Hong Kong SAR, China, ${ }^{4}$ Department of Electrical and Electronic Engineering, The University of Hong Kong, Hong Kong, Hong Kong SAR, China, ${ }^{5}$ Department of Pathology, Li Ka Shing Faculty of Medicine, The University of Hong Kong. Hong Kong. Hong Kong SAR, China

Background: To better understand the different clinical phenotypes across the disease spectrum in patients with COVID-19 using an unsupervised machine learning clustering approach.

Materials and Methods: A population-based retrospective study was conducted utilizing demographics, clinical characteristics, comorbidities, and clinical outcomes of 7,606 COVID-19-positive patients on admission to public hospitals in Hong Kong in the year 2020. An unsupervised machine learning clustering was used to explore this large cohort.

Results: Four clusters of differing clinical phenotypes based on data at initial admission was derived in which $86.6 \%$ of the deceased cases were aggregated in one of the clusters without prior knowledge of their clinical outcomes. Other distinctive clinical characteristics of this cluster were old age and high concurrent comorbidities as well as laboratory characteristics of lower hemoglobin/hematocrit levels, higher neutrophil, C-reactive protein, lactate dehydrogenase, and creatinine levels. The clinical patterns captured by the cluster analysis was validated on other temporally distinct cohorts in 2021. The phenotypes aligned with existing literature.

Conclusion: The study demonstrated the usefulness of unsupervised machine learning techniques with the potential to uncover latent clinical phenotypes. It could serve as a more robust classification for patient triaging and patient-tailored treatment strategies.

Keywords: COVID-19, clinical phenotypes, laboratory test, machine learning, clustering 


\section{INTRODUCTION}

Coronavirus disease 2019 (COVID-19) is a respiratory disease caused by severe acute respiratory syndrome coronavirus (SARS$\mathrm{CoV}-2$ ). This novel coronavirus was first reported in Wuhan, China, in December 2019 and quickly spread worldwide (1). Belonging to the same coronavirus family as SARS-CoV and MERS-CoV, SARS-CoV-2 also exhibits a remarkable infectivity power (2). Immediate practices have been taken to allocate medical resources and plan for treatments. However, they might not be as effective as expected due to a lack of knowledge about SARS-CoV-2. A better understanding of the pathogenesis of COVID-19 and its different clinical phenotypes and risk groups is essential to address the immunopathology of the infection. The accumulated observational data on COVID-19 positive patients available to date serve as valuable resources. To probe this large amount of clinical data, supervised machine learning approaches have been applied to the diagnosis and prognosis of COVID19 , risk stratification, and the prediction of different outcomes (3-9). Unsupervised machine learning is an alternative approach that does not require specific labels. It avoids using preconceived knowledge or assumptions that may be subjected to biases and unknown confounders. To this end, unsupervised clustering techniques are often used for exploratory analysis to probe the underlying patterns within big data sets, enabling identification of latent clinical phenotypes and potentially deriving novel insights from the associated correlations. For example, it has been applied to derive the phenotypes of COVID-19 patients using electronic health record (EHR) data on admission of 6,000 COVID-19-positive adults at the Mount Sinai Health System in New York in the United States (10), 413 patients from an individual-level published study (11), and 213 patients in Wuhan Pulmonary Hospital (12). Hong Kong's health system is unique when dealing with the COVID-19 pandemic. Due to a government-wide policy, all COVID-19-positive patients were admitted to public hospitals regardless of their disease severity or symptoms. Therefore, the data in Hong Kong can capture the varying presentations of COVID-19. This study aims to use unsupervised clustering analysis to discover different phenotypic presentations across the disease spectrum of COVID-19 in Hong Kong based on demographics and laboratory information on admission to hospital.

\section{MATERIALS AND METHODS}

\section{Study Design and Participants}

This study protocol was approved by institutional review boards in multiple hospitals across Hong Kong (see Supplementary Material for further details). Patient-informed consent was waived owing to the retrospective nature.

A retrospective search of patients' electronic records was conducted using the Hong Kong Hospital Authority Clinical Data Analysis and Reporting System (CDARS). It covered 42 hospitals across the territory in Hong Kong. Patients who were retrieved had a positive diagnosis of COVID-19 based on a reverse-transcriptase polymerase chain reaction (RT-PCR) test for SARS-CoV-2 fulfilling the testing criteria set by the Center for Health Protection, Department of Health, Government of Hong Kong SAR. The first cohort was retrieved from January 23 to December 31, 2020. The second cohort (used as a temporal validation set) was retrieved from January 1 to February 15, 2021.

Observational data, including demographics (age and sex), and 20 basic laboratory tests [white blood cell count (WBC), neutrophil count (NEUT), lymphocyte count (LYM), monocyte count (MON), hemoglobin (HGB), hematocrit (HCT), platelet (PLT), albumin (Alb), total bilirubin (TBIL), alanine aminotransferase (ALT), alkaline phosphatase (ALP), lactate dehydrogenase $(\mathrm{LDH})$, creatine kinase $(\mathrm{CK})$, urea, creatinine $(\mathrm{Cr})$, C-reactive protein $(\mathrm{CRP})$, sodium $(\mathrm{Na})$, potassium $(\mathrm{K})$, phosphate $(\mathrm{P})$, and calcium $(\mathrm{Ca})$ ] were retrieved on the first day of admission (see Table 1). Blood tests with $<50 \%$ of available data were excluded (13). Patients' comorbidities (19 systems) as specified by the international classification of disease (ICD-9) (14) were retrieved up to 3 days before that individual's admission to avoid including input of coding for the current admission. Mortality data was retrieved and set at 45 days after discharge for each patient to ensure that death, if it occurred, was likely related to COVID-19 and not from other causes. For more detailed information, please refer to Supplementary Table 1.

\section{Data Preparation and Preprocessing}

Yeo-Johnson transformation was applied to provide multivariate normal distributions (15). Patients with more than $40 \%$ missing variables and inconsistent data were excluded (13). Multiple imputation via chained equations (MICE) (16) was adopted to handle missing data and produced the least biased estimation under the verified assumption missing at random (MAR) (17). Bayesian ridge regression was used to introduce variations. Ten iterations and ascending order of imputations were set and deemed to be sufficient (18).

Principal components analysis (PCA) was used to compress intrinsically correlated and dependent numerical variables and project them to low-dimension representations (see Supplementary Figures 1, 2). Ten principal components (PCs) were kept to preserve $80 \%$ of the variance (see Supplementary Figure 3).

\section{Model Training}

$k$-prototype clustering (19) accounted for numerical data, and categorical data were used to probe the underlying clinical patterns of COVID-19-positive patients on admission. To select the number of clusters, the within-cluster sum of squared error (WCSS) was plotted from 1 to 10 clusters (see Supplementary Figure 4). According to the elbow method, four clusters were selected for the $k$-prototype model. The partitioning of three and five clusters was also examined (see Supplementary Figure 5).

\section{Model Evaluation}

To evaluate the generated clusters, a surrogate prediction model was built to check if partitioning found by the $k$-prototype model was still preserved. A gradient-boosting decision tree model named LightGBM (20) was used to build the prediction model. It predicted COVID-19 prognosis based on electronic 
TABLE 1 | Demographics and clinical characteristics of 7,606 COVID-19 positive patients.

\begin{tabular}{|c|c|c|}
\hline $\begin{array}{l}\text { Patients characteristics } \\
(n=7,606)\end{array}$ & $\begin{array}{l}\text { Median (IQR) } \\
\text { or count }(\%)^{*}\end{array}$ & $\begin{array}{l}\text { Missing } \\
\text { count }(\%)\end{array}$ \\
\hline \multicolumn{3}{|l|}{ I. Demographics } \\
\hline Age (years) & $47(32-61)$ & $0(0 \%)$ \\
\hline Sex (Males) & 3,697 (48.6\%) & $0(0 \%)$ \\
\hline \multicolumn{3}{|l|}{ II. Complete blood count } \\
\hline White blood cell count $\left({ }^{\star} 10^{9} / \mathrm{L}\right)$ & $5.3(4.3-6.6)$ & $0(0 \%)$ \\
\hline Neutrophil count $\left({ }^{*} 10^{9} / \mathrm{L}\right)$ & $3.2(2.4-4.3)$ & $110(1.4 \%)$ \\
\hline Lymphocyte count $\left({ }^{*} 10^{9} / \mathrm{L}\right)$ & $1.3(1.0-1.8)$ & $110(1.4 \%)$ \\
\hline Monocyte count $\left({ }^{*} 10^{9} / \mathrm{L}\right)$ & $0.5(0.4-0.7)$ & $110(1.4 \%)$ \\
\hline Hemoglobin (g/dL) & $13.7(12.6-14.7)$ & $0(0 \%)$ \\
\hline Hematocrit (L/L) & $0.40(0.37-0.43)$ & $1(<0.1 \%)$ \\
\hline Platelet $\left({ }^{*} 10^{9} / \mathrm{L}\right)$ & $214(173-264)$ & $5(<0.1 \%)$ \\
\hline \multicolumn{3}{|l|}{ III. Liver function } \\
\hline Albumin (g/L) & $40.0(37.2-43.5)$ & $3(<0.1 \%)$ \\
\hline Total bilirubin ( $\mu \mathrm{mol} / \mathrm{L})$ & $7.9(5.8-10.9)$ & $8(<0.1 \%)$ \\
\hline Alanine aminotransferase $(\mu / L)$ & $23.4(16.0-36.0)$ & $8(<0.1 \%)$ \\
\hline Alkaline phosphatase $(\mu / L)$ & $66(54-81)$ & $8(<0.1 \%)$ \\
\hline \multicolumn{3}{|l|}{ IV. Kidney function } \\
\hline Urea (mmol/L) & $3.9(3.1-4.8)$ & $5(<0.1 \%)$ \\
\hline Creatinine ( $\mu \mathrm{mol} / \mathrm{L})$ & $69.1(58.0-83.0)$ & $5(<0.1 \%)$ \\
\hline \multicolumn{3}{|l|}{ V. Inflammatory marker } \\
\hline C-reactive protein & $0.4(0.1-1.5)$ & 872 (11.5\%) \\
\hline \multicolumn{3}{|l|}{ VI. Electrolyte } \\
\hline Sodium (mmol/L) & $138(137-140)$ & $5(<0.1 \%)$ \\
\hline Potassium (mmol/L) & $3.8(3.5-4.1)$ & $31(0.4 \%)$ \\
\hline Phosphate (mmol/L) & $1.04(0.90-1.18)$ & $2,778(36.5 \%)$ \\
\hline Calcium (mmol/L) & $2.26(2.18-2.34)$ & $2,720(35.8 \%)$ \\
\hline \multicolumn{3}{|l|}{ VII. Others } \\
\hline Lactate dehydrogenase $(\mu / L)$ & $193.0(165.0-235.0)$ & 238 (3.1\%) \\
\hline Creatine kinase $(\mu / L)$ & $91(63-143)$ & 818 (10.8\%) \\
\hline
\end{tabular}

*Decimal places were kept according to normal reference range.

patient record data, and the performance was demonstrated (8). Eighty percent of features were used before training each decision tree to prevent overfitting. Missing data were ignored in the training. A separate cohort of 722 COVID-19 positive patients admitted from January 1 to February 15, 2021, were retrieved as a separate temporal validation set to assess the prediction (see Supplementary Table 2). Shapley additive explanation (SHAP) (21) was used to explain the feature importance of the prediction model. The data processing was conducted using Python version 3.8.5 (Python Software Foundation, Beaverton, USA) and available libraries.

\section{Statistical Analysis}

Descriptive statistics were generated using SPSS version 26 (IBM Corp, Armonk, NY). Because the numerical data were not normally distributed, medians and interquartile ranges were reported. For categorical data, counts and percentages were reported. The level of missing data was also reported.

To compare the intercluster dissimilarity of numerical data, the central limit theorem (CLT) was applied, and parametric ANOVA was conducted. A $p<0.05$ equates to statistical significance. Because the Levene's test of equality of error variances was statistically significant, equal variance across clusters was not assumed. Games-Howell post-hoc tests were performed for multiple comparisons. To compare the intercluster dissimilarity of categorical data, a chi-square test was conducted. Bonferroni post-hoc tests were performed for multiple comparisons. Interquartile range (IQR) and charts were reported and used for interpretation (22).

\section{RESULTS}

Initial data retrieval yielded a total of 8,562 patients. After data preparation to deal with missing variables and inconsistent data, a final number of 7,606 patients was kept (see Figure 1). The demographics and clinical characteristics are shown in Table $\mathbf{1 .}$

Four clusters were identified using demographics and laboratory variables on admission. A separate analysis was done on the deceased cohort (see Table 2). The clusters were compared with one another. Each cluster was compared with the derived population and the most used normal reference range in the cohort. Each cluster was also compared with the deceased cases. A value was marked "high/low" if still within the reference range but considered close to the cutoff values. A value was marked "elevated/reduced" if it was outside the normal reference range (see Table 2, Figure 2; Supplementary Table 3).

\section{General Characteristics}

The size of the four clusters were cluster $1: n=1,959$, cluster 2 : $n=2,224$, cluster $3: n=1,850$, and cluster $4: n=1,573$. Clusters 1 and 2 had the youngest median age [cluster 1: 36 (IQR: 24-50), cluster 2: 38 (IQR: 27-53)]. Increasing median age was observed from cluster 1 to 4 . Clusters 2 and 4 had a greater proportion of males [cluster $2: n=1,947$ (87.5\%), cluster $4: n=1,023(65.0 \%)$ ]. The lymphocyte count was in the low value of the reference range in four clusters [cluster 1: 1.7 (IQR: 1.3-2.2), cluster 2: 1.5 (IQR: 1.2-2.0), cluster 3: 1.1 (IQR: 0.8-1.3), cluster 4: 1.0 (IQR: 0.7-1.3), normal reference range: 1.0-3.1]. Most of the indicators for liver function, including total bilirubin and alanine phosphatase levels, and for electrolytes, including sodium and potassium, were in the normal reference range for four clusters. Specific cluster analysis was discussed as follows.

\section{Cluster 1}

Of COVID-19-positive patients, 25.4\% $(n=1,959)$ were aggregated in cluster 1 . Cluster 1 was characterized by the youngest median age and the highest proportion of females $(85.5 \%)$ within the cluster. The laboratory test results were unremarkable.

\section{Cluster 2}

Of COVID-19-positive patients, 28.9\% $(n=2,224)$ were aggregated in cluster 2 . Cluster 2 was characterized as the largest cluster with the highest proportion of males (87.5\%) within the cluster. Hemoglobin, hematocrit, and creatinine levels were observed in the higher range of the reference values. 


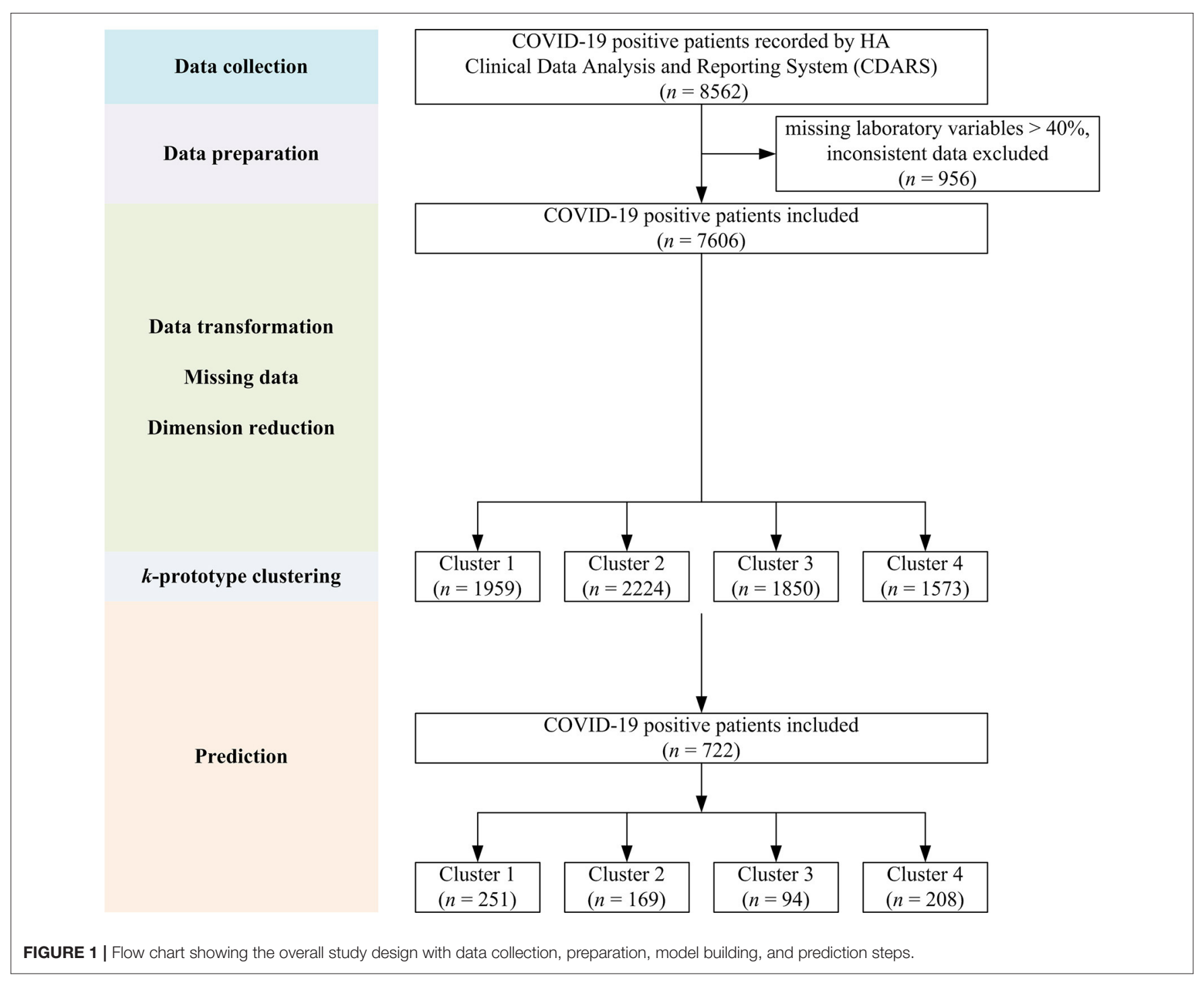

\section{Cluster 3}

Of COVID-19-positive patients, 24.0\% $(n=1,850)$ were aggregated in cluster 3 . Cluster 3 was characterized by a higher proportion of females (76.1\%) within the cluster. White blood cell counts were observed in the lower range of the reference values.

\section{Cluster 4}

Of COVID-19-positive patients, 20.4\% $(n=1,573)$ were grouped in cluster 4 . Significantly, cluster 4 captured 123 out of 142 (86.6\%) deceased cases. Cluster 4 was characterized by being the smallest cluster with the oldest median age and a higher proportion of males. In terms of blood tests, hemoglobin, hematocrit, lymphocyte, and albumin levels were observed in the lower range. Neutrophil, urea, and C-reactive protein were observed in the higher range of the reference values. Lactate dehydrogenase was elevated. Cluster 4 also had the highest comorbidity scores with triple the rate of immunity disorders and diseases of the circulatory systems; double the rate of diseases of the nervous systems; and a higher proportion of disease of the digestive system, genitourinary system, skin and subcutaneous tissue musculoskeletal system, other symptoms, injuries, and morphology of neoplasms compared with the other clusters. Cluster 4 captured almost all clinical characteristics of the deceased cases with the deceased cases having an even older median age and higher comorbidity scores.

\section{Deceased Cohort}

There were 142 deceased cases in 7,606 censored COVID-19positive patients. The deceased cohort was characterized by an old median age and a higher proportion of males. In terms of blood tests, lymphocyte, platelet, and albumin were observed in the lower range of the reference values. Urea and creatinine were observed in the higher range of the reference value. C-reactive protein and lactate dehydrogenase, although still in the reference range, were comparable to that of cluster 4 appearing higher than the other clusters. The comorbidity scores of the deceased cohort were high. There was a high degree of concordance with cluster 4 . 
TABLE 2 | Demographics and clinical characteristics of four clusters and deceased cohort for comparison.

$\begin{array}{lcccccc}\begin{array}{l}\text { Characteristics } \\ \left.\text { (unit; normal reference range }{ }^{\star \star}\right)\end{array} & \text { Cluster } 1 & \text { Cluster } 2 & \text { Cluster 3 } & \text { Cluster 4 } & p \text {-value } & \text { Deceased } \\ (n=1,959) & (n=2,224) & (n=1,850) & (n=1,573) & \text { cohort }\end{array}$

I. Demographics
Age

(years)

Sex

(Males)

II. Complete blood count

$\left({ }^{*} 10^{9} / \mathrm{L} ; 3.7-9.2\right)$

Neutrophil count

$\left({ }^{*} 10^{9} / \mathrm{L} ; 1.7-5.8\right)$

Lymphocyte count

( $\left.{ }^{*} 10^{9} / \mathrm{L} ; 1.0-3.1\right)$

Monocyte count

$\left({ }^{*} 10^{9} / \mathrm{L} ; 0.1-0.8\right)$

Hemoglobin

(g/dL; 11.7-14.9)

Hematocrit

(L/L; 0.35-0.45)

III. Liver function

\section{Albumin}

(g/L; 35.0-52.0)

Total bilirubin

( $\mu \mathrm{mol} / \mathrm{L} ; 5.0-21.0$ )

Alanine aminotransferase

( $\mu / L ; 0.0-34.4$ )

Alkaline phosphatase

( $\mu / L ; 30-120)$

Urea

(mmol/L; 2.8-8.1)

Creatinine

( $\mu \mathrm{mol} / \mathrm{L} ;$ 49.0-90.0)

v. Inflammatory marker

C-reactive protein

(mg/dL; 0.0-5.0)

\section{Electrolyte}

\section{Sodium}

(mmol/L; 136-145)

Potassium

(mmol/L; 3.4-4.8)

Phosphate

Calcium

(mmol/L; 2.15-2.55)

VII. Others

Lactate dehydrogenase ( $\mu / L ; 0.0-246.4)$

( $\mu / L ; 39-308)$
White blood Cell count

\section{Kidney function}

(mmol/L; 0.88-1.45)

Creatine kinase

$\begin{array}{cc}36 \mathrm{a}^{\star \star \star} & 38 \mathrm{~b} \\ (24-50) & (27-53) \\ 285 \mathrm{a} & 1947 \mathrm{~b} \\ (14.5 \%) & (87.5 \%)\end{array}$

Median (IQR) or count (within cluster \%)

$6.2 \mathrm{a}$
$(5.2-7.5)$
$3.7 \mathrm{a}$
$(2.9-4.8)$
$1.7 \mathrm{a}$
$(1.3-2.2)$
$0.5 \mathrm{a}$
$(0.4-0.7)$
$13.0 \mathrm{a}$
$(12.1-13.6)$
$0.38 \mathrm{a}$
$(0.36-0.40)$

\section{$5.6 \mathrm{~b}$}

(4.7-6.8)

$3.3 \mathrm{~b}$

(2.6-4.2)

$1.5 \mathrm{~b}$

$(1.2-2.0)$

$0.6 \mathrm{~b}$

(0.4-0.7)

$15.1 \mathrm{~b}$

(14.5-15.7)

$0.44 \mathrm{~b}$

$(0.43-0.46)$

$\begin{array}{cc}41.2 \mathrm{a} & 43.0 \mathrm{~b} \\ (38.8-43.9) & (40.9-45.5) \\ 7.0 \mathrm{a} & 9.5 \mathrm{~b} \\ (5.0-9.7) & (7.0-12.9) \\ 18.0 \mathrm{a} & 29.0 \mathrm{~b} \\ (13.1-27.0) & (20.0-45.0) \\ 67 \mathrm{a} & 69 \mathrm{~b} \\ (54-87) & (58-82)\end{array}$

\section{$3.4 \mathrm{a}$}

(2.7-4.1)

$57.0 \mathrm{a}$

(50.8-64.0)

$$
\begin{gathered}
4.2 \mathrm{~b} \\
(3.6-5.0) \\
80.0 \mathrm{~b} \\
(72.0-90.0)
\end{gathered}
$$

\section{$0.2 \mathrm{a}$}

(0.1-0.4)

$$
0.3 \mathrm{~b}
$$

(0.1-0.7)

$$
\begin{gathered}
139 a \\
(137-140) \\
3.8 \mathrm{a} \\
(3.5-4.0) \\
1.11 \mathrm{a} \\
(0.98-1.27) \\
2.31 \mathrm{a} \\
(2.25-2.38)
\end{gathered}
$$

$$
\begin{gathered}
139 a \\
(138-140) \\
3.9 \mathrm{~b} \\
(3.6-4.1) \\
1.07 \mathrm{~b} \\
(0.95-1.20) \\
2.33 \mathrm{a} \\
(2.26-2.39)
\end{gathered}
$$

$176.5 \mathrm{a}$
$(154.0-208.0)$
$67 a$

182.2b

(161.0-210.0)

$104 \mathrm{~b}$

(49-91)

$51 \mathrm{c}$
$(38-61)$
$442 \mathrm{c}$
$(23.9 \%)$

$3.8 \mathrm{c}$
$(3.2-4.3)$
$2.2 \mathrm{c}$
$(1.7-2.7)$
$1.1 \mathrm{c}$
$(0.8-1.3)$
$0.4 \mathrm{c}$
$(0.3-0.5)$
$13.2 \mathrm{c}$
$(12.3-13.9)$
$0.39 \mathrm{c}$
$(0.37-0.41)$

$65 d$
$(57-75)$
$1023 d$
$(65.0 \%)$

$5.8 \mathrm{~d}$

(4.8-7.1)

$4.0 d$

(3.2-5.3)

$1.0 \mathrm{~d}$

(0.7-1.3)

$0.5 \mathrm{a}$

(0.4-0.7)

$13.3 \mathrm{c}$

(12.1-14.2)

$0.39 \mathrm{c}$

(0.36-0.42)
40.0c
(37.0-42.0)
$6.6 \mathrm{c}$
(5.0-9.0)
$20.0 \mathrm{a}$
(14.0-28.5)
$60 \mathrm{c}$
(50-73)
36.0d
(32.0-39.0)
$8.2 \mathrm{~d}$
(6.2-11.3)
$29.0 \mathrm{~b}$
(20.0-44.0)
$67 \mathrm{~b}$
(56-84)

$3.6 \mathrm{c}$
$(2.9-4.3)$
$72.0 \mathrm{c}$

(62.8-84.0)

$4.9 d$
$(3.9-6.6)$
$82.0 d$

(69.0-101.0)

$$
\begin{gathered}
0.5 \mathrm{c} \\
(0.2-1.2)
\end{gathered}
$$

$3.6 \mathrm{~d}$

(1.6-7.2)

$139 b$
$(137-140)$
$3.7 c$

$136 \mathrm{c}$
$(133-138)$

$3.8 \mathrm{a}$

(3.5-4.1)

$0.95 d$

$1.02 \mathrm{C}$

(0.89-1.15)

$2.22 \mathrm{~b}$

(2.16-2.28)

(0.83-1.09)

$2.17 \mathrm{c}$

(2.10-2.24)
$191.0 \mathrm{c}$
(164.0-220.0)
$82 \mathrm{c}$

(59-120)

(76-151)
263.0d
(212.0-350.0)
$141 d$

(85-258)
$<0.001$

81

(74-87)

87

(61.3\%)

$\begin{array}{cc}<0.001 & 6.9 \\ & (5.1-9.0) \\ <0.001 & 4.9 \\ & (3.5-7.4) \\ <0.001 & 1.0 \\ & (0.7-1.3) \\ <0.001 & 0.6 \\ & (0.3-0.8) \\ <0.001 & 12.2 \\ & (10.7-13.4) \\ <0.001 & 0.36 \\ & (0.32-0.40)\end{array}$

$<0.001$

34.0

8.2

(6.0-11.6)

20.8

(14.0-33.2)

72

(59-103)

$<0.001$

7.0

(5.3-10.0)

101.1

(73.0-137.7)

$<0.001$

3.6

(1.3-8.6)

$<0.001$

136

(133-140)

$<0.001$

4.0

$<0.001$

1.08

(0.90-1.23)

$<0.001$

2.14

$(2.06-2.25)$
249.0

(199.1-378.8)

$<0.001 \quad 131$

$(71-250)$
(29.0-38.1)

$(3.7-4.3)$

${ }^{\star \star}$ Most used normal reference range.

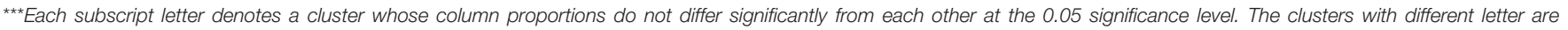
significantly different from each other at the 0.05 significance level. 


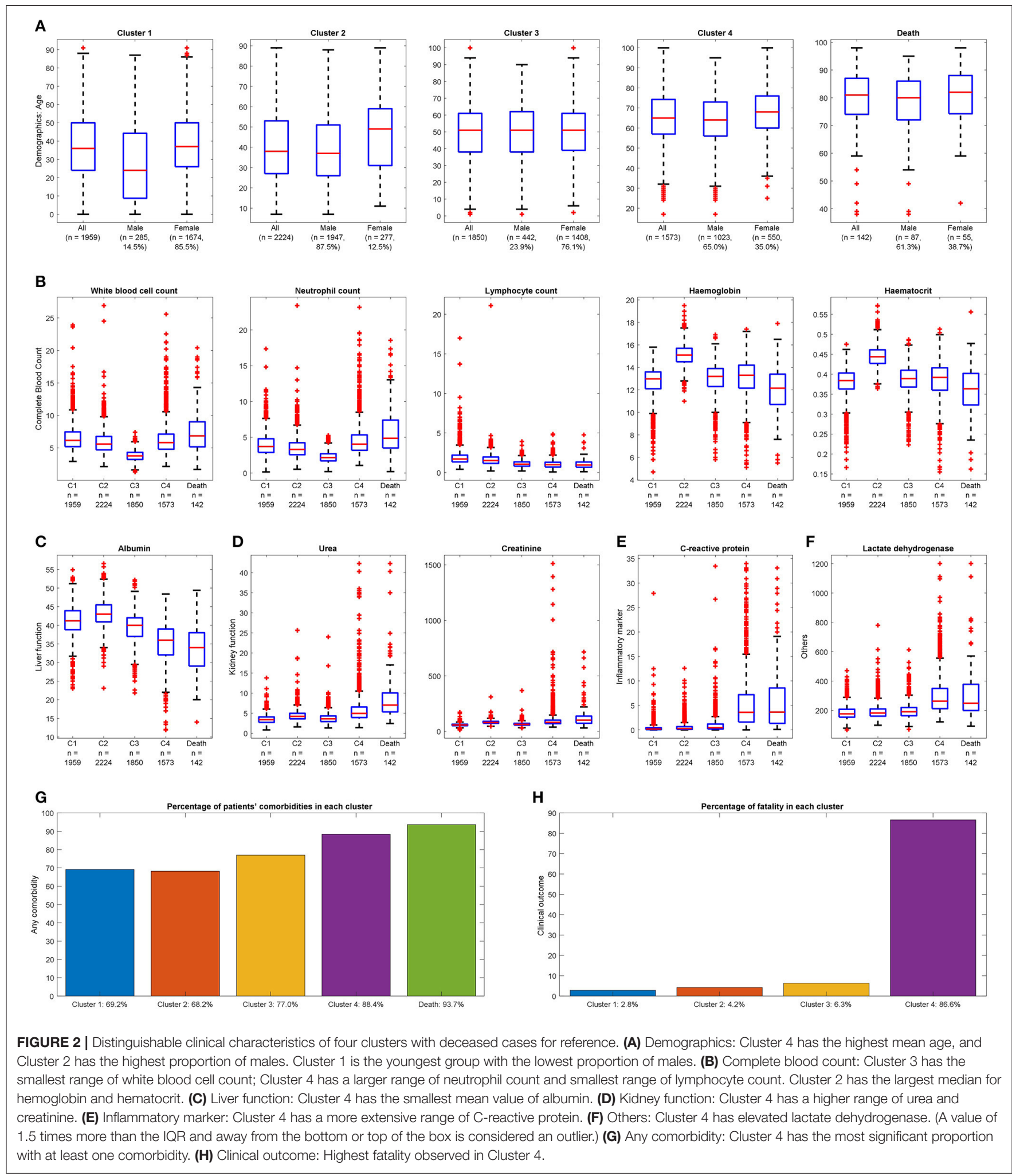

\section{Evaluation}

Cluster analysis was also applied to a separate temporal validation set with the results of seven out of eight (87.5\%) deceased cases being captured by cluster 4 .
To further verify if criteria generated by $k$-prototype clustering were properly followed, SHAP was used to determine the feature importance used in the classification (see Figure 3). Overall, white blood count has the highest mean SHAP value for the 

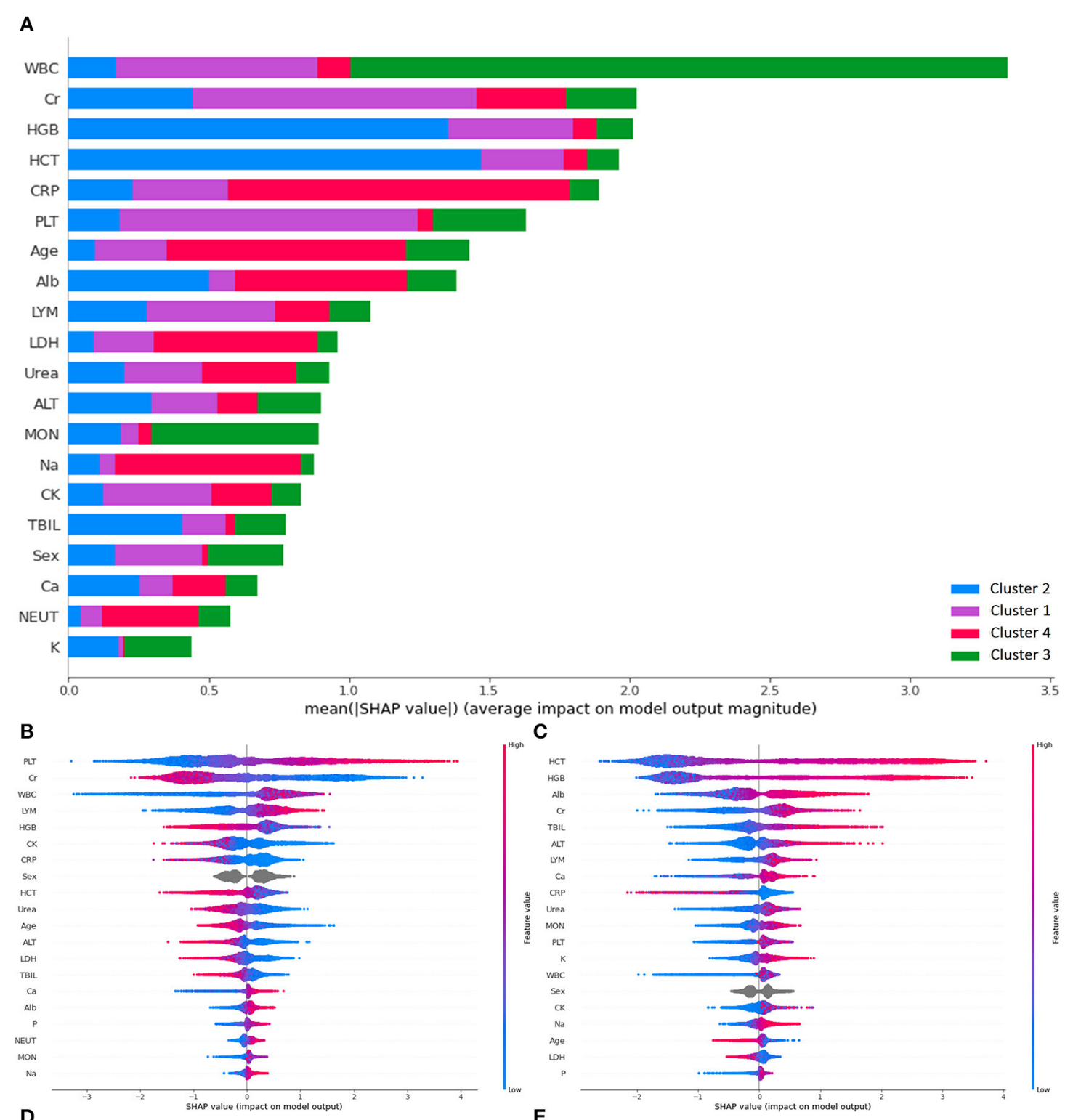

D
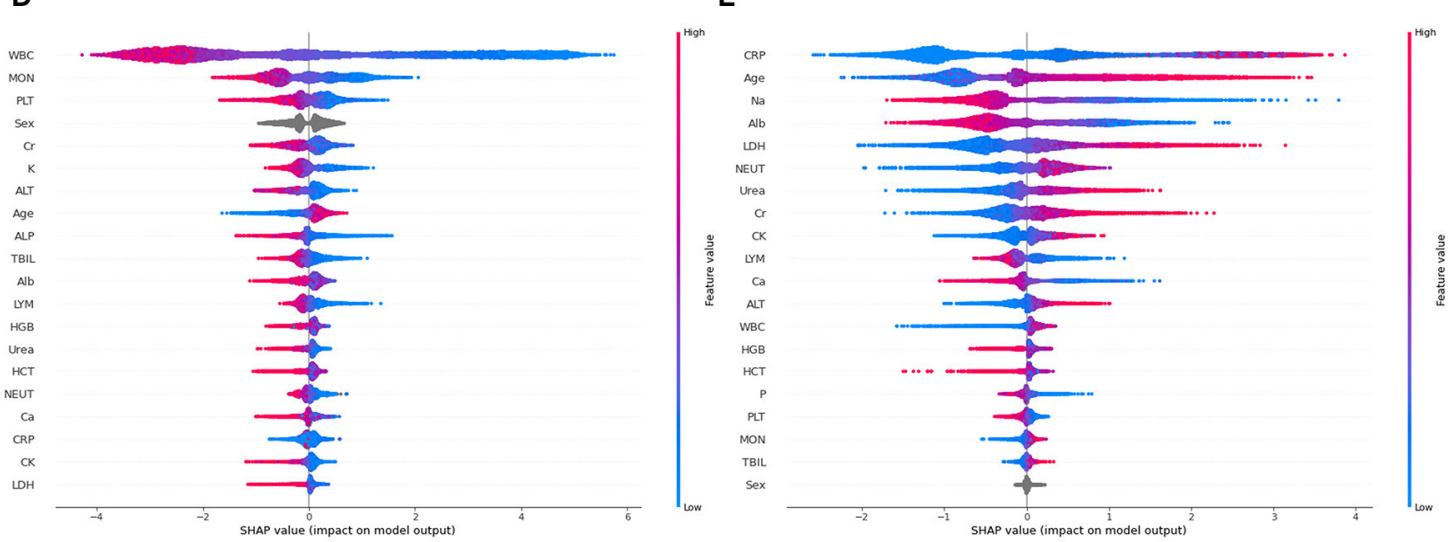

FIGURE 3 | SHAP plots demonstrating differential importance of different features and clusters. (A) Mean SHAP value of the prediction, (B) SHAP value for cluster 1 , (C) SHAP value for cluster 2, (D) SHAP value for cluster 3, (E) SHAP value for cluster 4. ALT, Alanine aminotransferase; Alb, albumin; ALP, alkaline phosphatase; Ca, calcium; CRP, C-reactive protein; CK, creatine kinase; Cr, creatinine; HCT, hematocrit; HGB, hemoglobin; LDH, lactate dehydrogenase; LYM, lymphocyte count; MON, monocyte count; NEUT, neutrophil count; P, phosphate; PLT, platelet; K, potassium; Na, sodium; TBIL, total bilirubin; WBC, white blood cell. 
prediction, followed by creatinine, hemoglobin, hematocrit, Creactive protein, and platelet. High platelet and low creatinine were most important in classifying patients into cluster 1 . Elevated hemoglobin and hematocrit were most important in classifying patients into cluster 2 . Reduced white blood cell count was the most important in classifying patients into cluster 3 . High C-reactive protein and old age were most important in classifying patients into cluster 4 .

\section{DISCUSSION}

Unsupervised clustering was used to probe the latent phenotypes of 7,606 COVID-19-positive patients in Hong Kong across 2020. Based on age, sex, and 20 laboratory blood tests on admission, one of the four generated clusters aggregated $86.6 \%$ of deceased cases without prior information of their clinical outcomes. The clinical characteristics of this cluster, including the oldest median age; highest aggregated comorbidity; and the laboratory tests of hemoglobin, hematrocrit, and lymphocyte were observed to be in the lower range of normal, whereas neutrophil, Creactive protein, and lactate dehydrogenase were observed to be in the higher range of normal. Findings are comparable with poor prognostic features based on contemporaneous literature. Notably, when applied to a separate validation cohort, cluster 4 was still able to identify seven out of eight (87.5\%) deceased cases. A potential clinical utility may be to call for early medical attention and resource to patients that belong to cluster 4 at the initial diagnosis at hospitalization. The clinical characteristics of cluster 4 and deceased cases in this study aligned with previous findings (23-25). First, old age reflects a weaker immune system to fight against pathogens, including pneumonia (26). It is a well-established risk factor correlated with chronicity and comorbidity in COVID-19 prognosis $(10,27)$. On the other hand, increased neutrophil count and high neutrophil-tolymphocyte ratio can be elicited by deep airway and alveolar damage. They represent an acute inflammatory response and are indicators of a poor prognosis and higher disease severity for COVID-19 (28-31). In line with cluster 4 and the deceased cases in this study, at an early stage of COVID-19, lymphocyte counts typically decrease, whereas white blood cell count may or may not decrease (32). In addition, patients with severe COVID-19 outcomes have exhibited abnormally high C-reactive protein levels, lactate dehydrogenase, and neutrophils, implying an inflammatory response and severe pneumonia (33). Although C-reactive protein level is not affected by physical status, age, and sex (34), it may be used to early diagnose severe pulmonary disease secondary to bacterial infection (35).

A greater proportion of males than females were infected with COVID-19 in contemporary literatures as well as MERS-CoV and SARS-CoV infection $(24,36,37)$. It can be a cofounder in evaluating the sex-based difference in the susceptibility of COVID-19 (38). In this retrospective study, the proportion of males and females was roughly equal across all age groups (see Figure 4) on admission. No significant difference in disease prevalence existed between males and females. Under this condition, a higher proportion of males was still observed in

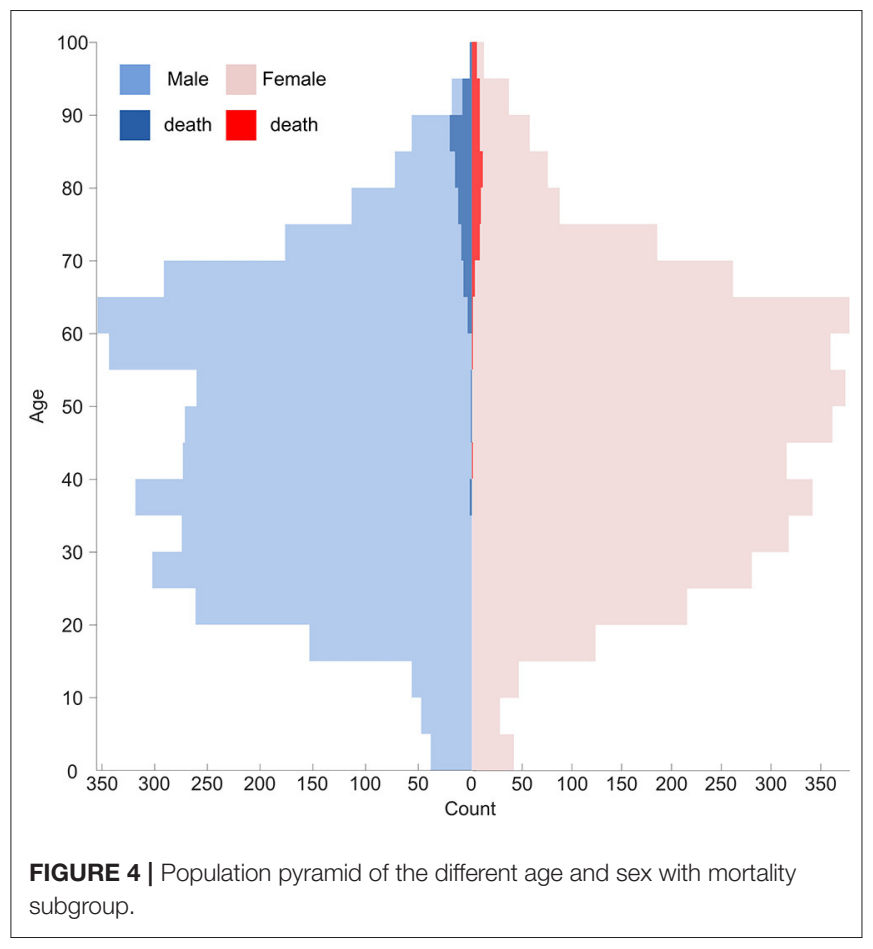

cluster $4(65.0 \%)$ and deceased cases $(61.3 \%)$. It indicates being male as a risk factor for mortality $(37,39,40)$. This reduced disease susceptibility of females is hypothesized to be related to the major roles of the $\mathrm{X}$ chromosome and sex hormones in innate and adaptive immunity (41). Nonetheless, this correlation needs further exploration and investigation.

The complications of COVID-19 include severe pneumonia, septic shock, acute respiratory distress syndrome (ARDS), and multiple organ failure $(31,42)$. Although lung has been the primary organ involved in the infection, elevated creatinine and urea levels were also detected in cluster 4 and in the deceased cases, which implies that impaired renal function was a potential early indicator of poor prognosis. Additionally, a low albumin level, reflective of liver synthetic function, was also observed. These abnormal laboratory variables imply that indicators for early multiorgan failure were important and is likely a reflection of systemic inflammatory response. For the reduced hemoglobin and hematocrit levels detected in cluster 4 and the deceased, a prior study suggests that SARS-CoV-2 may attack the heme on 1-beta chain of hemoglobin through CD147, and CD26, ACE2 receptors or by simulating hepcidin to increase tissue ferritin, block ferroprotein, causing the iron deficiency, and thus lower hemoglobin level (43).

In previous clustering analysis (10-12), both three and four clusters have been identified, which were similar to the number chosen by this study. Previous clusters have also found some correlations for a poor prognosis, such as old age and high comorbidity scores $(12,28)$, being male, high lymphocytes high neutrophil count (11), and albumin level (12). In respect to the clinical outcomes, our study shows the greatest power to differentiate a cluster by aggregating $86.6 \%$ 
deceased cases and capturing most of their clinical characteristics and correlations.

There were several key strengths to our study. First, a large sample size of 7,606 COVID-19-positive patients was included. This adds to greater statistical power and generalizability. The data were collected from a single integrated EHR system fully covering the Hong Kong population, meaning the clinical data set is representative of this cohort. Second, all patients that were tested positive despite mild or no symptoms were nevertheless hospitalized in Hong Kong. Our study is unique capturing a wide spectrum of clinical severity. On the contrary, most other countries only tend to admit patients with severe symptoms to the hospitals. Third, we validated our clusters on a separate temporal validation with further feature explanation using SHAP. It was demonstrated that criteria from PCA-based $k$ prototype clustering were correctly captured, used, and inferred. Granular analysis of these distinct clusters should give a better understanding of different clinical representations of COVID19 on admission. Besides this, delineating interactions between specific chronicity and poor prognosis regarding different clinical groups can help understand the mechanism of COVID-19.

There were a few limitations to our study. First, for a retrospective study using observational data, the sampling was not random. There may be the potential of selection and recall bias. Although all age groups were represented, they were not evenly distributed. The results and interpretations will be best applied to the Hong Kong cohort with race dominated by patients of Chinese ethnicity although there were several imported cases of different ethnic origins. Owing to a lack of accurate data on ethnicity, we could not perform further subanalysis. Second, as with any large-scale studies covering multiple hospitals, there may be different standards and measurement protocols. It was observed that the normal reference ranges of laboratory variables varied mildly across institutions and according to age and sex. Because no significant discrepancy was detected, the data were put together directly using their original values. Although a linear mapping could have been used to reduce the systematic noise, variations due to age and sex need to be further distinguished. Finally, some potential important clinical and laboratory data with more than $50 \%$ of missing data were excluded from the study. They include BMI, blood pressure, oxygen saturation, D-Dimer, need for assisted ventilation, ITU admission, etc., which are potentially useful for severity assessment and prognostication. These may additionally aid prediction and more accurate allocation of medical resources in the future. Owing to a lack of testing or

\section{REFERENCES}

1. Zhu N, Zhang D, Wang W, Li X, Yang B, Song J, et al. A Novel Coronavirus from Patients with Pneumonia in China, 2019. N Engl J Med. (2020) 382:72733. doi: 10.1056/NEJMoa2001017

2. Li Q, Guan X, Wu P, Wang X, Zhou L, Tong Y, et al. Early transmission dynamics in Wuhan, China, of novel coronavirus-infected pneumonia. $N$ Engl J Med. (2020) 382:1199-207. doi: 10.1056/NEJMoa2001316 accurate documentations, these were not able to be included in this large scale multi-institutional study. These variables are recommended to be documented or performed in the future. However, they were not routinely recorded nor taken in usual clinical practice in Hong Kong at the time of the study period.

\section{CONCLUSION}

Unsupervised clustering was used to probe the latent phenotypes of 7,606 COVID-19-positive patients in Hong Kong across the year 2020. Based on age, sex, and 20 laboratory variables on admission, one of the four generated clusters aggregated $86.6 \%$ deceased cases without prior knowledge of their clinical outcomes. Further understanding of the different COVID-19 clinical phenotypes may pave the way for more individualized patient risk stratification and treatment.

\section{DATA AVAILABILITY STATEMENT}

Data can be provided upon reasonable request and inquiries can be directed to the corresponding author

\section{ETHICS STATEMENT}

The studies involving human participants were reviewed and approved by Cluster Research Ethics Committee/Institutional Review Board (REC/IRB). Written informed consent for participation was not required for this study in accordance with the national legislation and the institutional requirements.

\section{AUTHOR CONTRIBUTIONS}

$\mathrm{KL}, \mathrm{K}-\mathrm{SN}$, and VV were involved in study conception, statistical analysis, and drafting of the initial version of the manuscript. $\mathrm{K}-\mathrm{SN}$ and $\mathrm{VV}$ were involved in data collection. K-WK, KT, C-FS, C-WL, and VV supervised the work and offered significant intellectual contribution. All authors discussed the data analysis, results, and approved the final version.

\section{SUPPLEMENTARY MATERIAL}

The Supplementary Material for this article can be found online at: https://www.frontiersin.org/articles/10.3389/fmed. 2021.764934/full\#supplementary-material machine learning-based model for survival prediction in patients with severe COVID-19 infection. medRxiv. (2020) 7:1-7. doi: 10.1101/2020.02.27.200 28027

4. Wynants L, Van Calster B, Collins GS, Riley RD, Heinze G, Schuit E, et al. Prediction models for diagnosis and prognosis of covid-19: systematic review and critical appraisal. BMJ. (2020) 369:m1328. doi: 10.1136/bmj. $\mathrm{m} 1328$ 
5. Vaid A, Somani S, Russak AJ, De Freitas JK, Chaudhry FF, Paranjpe I, et al. Machine learning to predict mortality and critical events in a cohort of patients with COVID-19 in New York City: model development and validation. J Med Internet Res. (2020) 22:e24018. doi: 10.2196/ 24018

6. Alakus TB, Turkoglu I. Comparison of deep learning approaches to predict COVID-19 infection. Chaos Soliton Fract. (2020) 140:110120. doi: 10.1016/j.chaos.2020.110120

7. An C, Lim H, Kim D-W, Chang JH, Choi YJ, Kim SW. Machine learning prediction for mortality of patients diagnosed with COVID-19: a nationwide Korean cohort study. Sci Rep. (2020) 10:18716. doi: 10.1038/s41598-020-75767-2

8. Ferrari D, Milic J, Tonelli R, Ghinelli F, Meschiari M, Volpi $\mathrm{S}$, et al. Machine learning in predicting respiratory failure in patients with COVID-19 pneumonia-Challenges, strengths, and opportunities in a global health emergency. PLoS ONE. (2020) 15:e0239172. doi: 10.1371/journal.pone.0239172

9. Du R, Tsougenis ED, Ho JWK, Chan JKY, Chiu KWH, Fang BXH, et al. Machine learning application for the prediction of SARS-CoV2 infection using blood tests and chest radiograph. Sci Rep. (2021) 11:14250. doi: 10.1038/s41598-021-93719-2

10. Cui W, Robins D, Finkelstein J. Unsupervised machine learning for the discovery of latent clusters in COVID-19 patients using electronic health records. Stud Health Technol Inform. (2020) 272:1-4. doi: 10.3233/SHTI200478

11. Li WT, Ma J, Shende N, Castaneda G, Chakladar J, Tsai JC, et al. Using machine learning of clinical data to diagnose COVID-19: a systematic review and meta-analysis. BMC Med Inform Decis Mak. (2020) 20:247. doi: 10.1186/s12911-020-01266-Z

12. Ye W, Lu W, Tang Y, Chen G, Li X, Ji C, et al. Identification of COVID-19 clinical phenotypes by principal component analysis-based cluster analysis. Front Med. (2020) 7:570614. doi: 10.3389/fmed.2020.570614

13. Jakobsen JC, Gluud C, Wetterslev J, Winkel P. When and how should multiple imputation be used for handling missing data in randomised clinical trials - a practical guide with flowcharts. BMC Med Res Methodol. (2017) 17:162. doi: 10.1186/s12874-017-0442-1

14. World Health Organisation. International Classification of Diseases: [9th] Ninth Revision, Basic Tabulation List With Alphabetic Index. Geneva: World Health Organization (1978).

15. Yeo IK, Johnson RA. A new family of power transformations to improve normality or symmetry. Biometrika. (2000) 87:9549. doi: 10.1093/biomet/87.4.954

16. Raghunathan TE, Lepkowski JM, Van Hoewyk J, Solenberger P. A multivariate technique for multiply imputing missing values using a sequence of regression models. Surv Methodol. (2001) 27:85-96.

17. Carpenter JR, Kenward MG. Missing Data in Randomised Controlled Trials: A Practical Guide. Birmingham: Health Technology Assessment Methodology Programme (2007). p. 199.

18. Papageorgiou G, Grant SW, Takkenberg JJM, Mokhles MM. Statistical primer: how to deal with missing data in scientific research? Interact CardioVasc Thoracic Surg. (2018) 27:153-8. doi: 10.1093/icvts/ivy102

19. Huang Z. Extensions to the k-means algorithm for clustering large data sets with categorical values. Data Min Knowl Discov. (1998) 2:283-304.

20. Ke G, Meng Q, Finley T, Wang T, Chen W, Ma W, et al. (editors). LightGBM: A Highly Efficient Gradient Boosting Decision Tree. Long Beach, CA: NIPS (2017).

21. Lundberg SM, Erion G, Chen H, DeGrave A, Prutkin JM, Nair B, et al. From local explanations to global understanding with explainable AI for trees. Nat Machine Intell. (2020) 2:56-67. doi: 10.1038/s42256-019-0138-9

22. Kaplan RM, Chambers DA, Glasgow RE. Big data and large sample size: a cautionary note on the potential for bias. Clin Transl Sci. (2014) 7:3426. doi: $10.1111 /$ cts. 12178

23. Wang D, Hu B, Hu C, Zhu F, Liu X, Zhang J, et al. Clinical characteristics of 138 hospitalized patients with 2019 novel coronavirus-infected pneumonia in Wuhan, China. JAMA. (2020) 323:1061-9. doi: 10.1001/jama.2020.1585

24. Chen N, Zhou M, Dong X, Qu J, Gong F, Han Y, et al. Epidemiological and clinical characteristics of 99 cases of 2019 novel coronavirus pneumonia in Wuhan, China: a descriptive study. Lancet. (2020) 395:507-13. doi: 10.1016/S0140-6736(20)30211-7

25. Guan W-j, Ni Z-y, Hu Y, Liang W-h, Ou C-q, He J-x, et al. Clinical characteristics of coronavirus disease 2019 in China. N Engl J Med. (2020) 382:1708-20. doi: 10.1056/NEJMoa2002032

26. Yang J, Zheng Y, Gou X, Pu K, Chen Z, Guo Q, et al. Prevalence of comorbidities and its effects in patients infected with SARS-CoV-2: a systematic review and meta-analysis. Int J Infect Dis. (2020) 94:915. doi: 10.1016/j.ijid.2020.03.017

27. Lee K-Y. Pneumonia, acute respiratory distress syndrome, and early immunemodulator therapy. Int J Mol Sci. (2017) 18:388. doi: 10.3390/ijms180 20388

28. Zhang B, Zhou X, Zhu C, Feng F, Qiu Y, Feng J, et al. Immune phenotyping based on neutrophil-to-lymphocyte ratio and IgG predicts disease severity and outcome for patients with COVID-19. medRxiv. (2020). doi: 10.1101/2020.03.12.20035048

29. Ye W, Chen G, Li X, Lan X, Ji C, Hou M, et al. Dynamic changes of D-dimer and neutrophil-lymphocyte count ratio as prognostic biomarkers in COVID-19. Respir Res. (2020) 21:169. doi: 10.1186/s12931-020-0 1428-7

30. Celikbilek M, Dogan S, Ozbakir O, Zararsiz G, Kücük H, Gürsoy S, et al. Neutrophil-lymphocyte ratio as a predictor of disease severity in ulcerative colitis. J Clin Lab Anal. (2013) 27:72-6. doi: 10.1002/jcla. 21564

31. Huang C, Wang Y, Li X, Ren L, Zhao J, Hu Y, et al. Clinical features of patients infected with 2019 novel coronavirus in Wuhan, China. Lancet. (2020) 395:497-506. doi: 10.1016/S0140-6736(20) 30183-5

32. Jin Y-H, Cai L, Cheng Z-S, Cheng H, Deng T, Fan Y-P, et al. A rapid advice guideline for the diagnosis and treatment of 2019 novel coronavirus (2019. $\mathrm{nCoV}$ ) infected pneumonia (standard version). Military Med Res. (2020) 7:4. doi: 10.1186/s40779-020-0233-6

33. Wang L. C-reactive protein levels in the early stage of COVID19. Méd Maladies Infect. (2020) 50:332-4. doi: 10.1016/j.medmal.2020. 03.007

34. Bilgir O, Bilgir F, Calan M, Calan OG, Yuksel A. Comparison of pre- and post-levothyroxine high-sensitivity c-reactive protein and fetuin-a levels in subclinical hypothyroidism. Clinics. (2015) 70:97-101. doi: 10.6061/clinics/2015(02)05

35. Warusevitane A, Karunatilake D, Sim J, Smith C, Roffe C. Early diagnosis of pneumonia in severe stroke: clinical features and the diagnostic role of C-reactive protein. PLoS ONE. (2016) 11:e0150269. doi: 10.1371/journal.pone.0150269

36. Badawi A, Ryoo SG. Prevalence of comorbidities in the Middle East respiratory syndrome coronavirus (MERS-CoV): a systematic review and meta-analysis. Int $J$ Infect Dis. (2016) 49:129-33. doi: 10.1016/j.ijid.2016.06.015

37. Channappanavar R, Fett C, Mack M, Ten Eyck PP, Meyerholz DK, Perlman S. Sex-based differences in susceptibility to severe acute respiratory syndrome coronavirus infection. J Immunol. (2017) 198:4046-53. doi: 10.4049/jimmunol.1601896

38. Docherty A, Harrison E, Green C, Hardwick H, Pius R, Norman L, et al. Features of 16,749 hospitalised UK patients with COVID-19 using the ISARIC WHO Clinical Characterisation Protocol. medRxiv. (2020). doi: 10.1101/2020.04.23.20076042

39. Deng G, Yin M, Chen X, Zeng F. Clinical determinants for fatality of 44,672 patients with COVID-19. Crit Care. (2020) 24:179. doi: 10.1186/s13054-020-02902-w

40. Williamson EJ, Walker AJ, Bhaskaran K, Bacon S, Bates C, Morton CE, et al. Factors associated with COVID-19-related death using OpenSAFELY. Nature. (2020) 584:430-6. doi: 10.1038/s41586-020-2521-4

41. Jaillon S, Berthenet K, Garlanda C. Sexual dimorphism in innate immunity. Clin Rev Allergy Immunol. (2019) 56:308-21. doi: 10.1007/s12016-0178648-x

42. Xu Z, Shi L, Wang Y, Zhang J, Huang L, Zhang C, et al. Pathological findings of COVID-19 associated with acute respiratory distress syndrome. Lancet Respir Med. (2020) 8:420-2. doi: 10.1016/S2213-2600(20)30076-X 
43. Cavezzi A, Troiani E, Corrao S. COVID-19: hemoglobin, iron, and hypoxia beyond inflammation. A narrative review. Clin Pract. (2020) 10:1271. doi: 10.4081/cp.202 0.1271

Conflict of Interest: The authors declare that the research was conducted in the absence of any commercial or financial relationships that could be construed as a potential conflict of interest.

Publisher's Note: All claims expressed in this article are solely those of the authors and do not necessarily represent those of their affiliated organizations, or those of the publisher, the editors and the reviewers. Any product that may be evaluated in this article, or claim that may be made by its manufacturer, is not guaranteed or endorsed by the publisher.

Copyright (c) $2022 \mathrm{Lau}, \mathrm{Ng}$, Kwok, Tsia, Sin, Lam and Vardhanabhuti. This is an open-access article distributed under the terms of the Creative Commons Attribution License (CC BY). The use, distribution or reproduction in other forums is permitted, provided the original author(s) and the copyright owner(s) are credited and that the original publication in this journal is cited, in accordance with accepted academic practice. No use, distribution or reproduction is permitted which does not comply with these terms. 